\title{
ESTUDIO PRELIMINAR DE LA RESISTENCIA DE Vibrio cholerae O1, BROTE EPIDÉMICO 1998, A ANTIMICROBIANOS
}

\section{PRELIMINARY SURVEY FROM THE ANTIMICROBIAL RESISTANCE OF Vibrio cholerae 01, EPIDEMIC OUTBREAK 1998}

\author{
J. O. Ibarra', L. E. Flores², R. H. García ${ }^{2}$ y D. E. Alvarado²
}

ABSTRACT

Thirty strains of $V$. cholerae 01 Ogawa and 2 Inaba, isolated during the outbreak of 1998 , were studied in relation to thier sensibility in front of 10 antibiotics of clinical use. Nine strains were determined $(28.1 \%)$ resistant to 1 up to 6 antibiotics. The SDS-PAGE of the studied strains exhibited similar pattern of bands.

La presencia de Vibrio cholerae $\mathrm{O} I$ en e] Perú tiene características endémicas, y la ocurrencia de la bacteria en nuestro medio obedece a factores ambientales, por lo que durante el Evento El Niño Oscilación Sur (ENOS) 1997 98 se incrementaron los casos de cólera (Colwell et. al., 1998; Ibarra et al., 1999). Generalmente, V. cholerae $O 1$ es sensible a los antimicrobianos utilizados en el tratamiento (Gotuzzo et al., 1994), pero se reporta su resistencia a partir de los años 70. En el Perú se han realizado estudios relacionados a la resistencia de la bacteria a antibióticos (Seto et al., 1992; Seas y Gotuzzo 1999), así como al compuesto vibriostático O/129 (Ibarra y Alvarado 1999).

El presente trabajo tiene por objetivo estudiar la evolución de la resistencia de cepas de $V$. cholerae $\mathrm{Ol}$ aisladas en los meses de verano de 1998, frente a antibióticos de uso clínico.

Se estudiaron 32 estirpes de $V$. cholerae OI, de las cuales 30 eran Ogawa y 2 Inaba, todas

1 Fac. Ciencias Naturales y Matemáticas, UNFV

2 Lab. Microbiologia Molecular. Fac. de C C. 8 B., UNMSM. Apido /20. Lima

E-mail jon171 Sholmail.com aisladas entre enero y marzo de 1998 y pertenecientes a la colección del Lab. de Microbiología Molecular de la Fac. de Ciencias Biológicas, UNMSM (Ibarra et al., 1999).

Se utilizó el método de Bauer y col. (Bauer et al., 1966). Se emplearon discos comerciales de Ampicilina (A)(10 $\mu \mathrm{g})$, Cloramfenicol (C)(30 $\mu \mathrm{g})$, Sulfatrimetoprim $(\mathrm{Sx})(25 \mu \mathrm{g})$, Tetraciclina $(\mathrm{T})(30 \mu \mathrm{g})$, Estreptomicina $(\mathrm{S})$ $(10 \mu \mathrm{g})$, Ácido Nalidíxico (M)(30 $\mu \mathrm{g})$, Furazolidone $(\mathrm{Fz})(100 \mu \mathrm{g})$, Ciprofloxacina (Cip) $(5 \mu g)$, Doxiciclina (Dxs)(30 $\mu g) y$ Eritromicina (EM) $(15 \mu \mathrm{g})$. Se midieron los halos de inhibición en milímetros y la interpretación se realizó según las normas establecidas por el NCCLS para Enterobacteriaceae (NCCLS 1992).

Los aislados fueron cultivados en $10 \mathrm{~mL}$ de Caldo Triptosa por $18 \mathrm{~h}$. Luego se centrifugaron a $4500 \mathrm{rpm}$ por 15 min suspendiéndose el sedimento en $1 \mathrm{~mL}$ de agua bidestilada. Se mezcló $100 \mu \mathrm{L}$ del cultivo con buffer de carga y se calentó en baño María por $10 \mathrm{~min}$. Las mezclas fueron sometidas a electroforesis en Gel de Poliacrilamida (SDS-PAGE) usando geles de 
12\%. Como marcador de corrida se utilizó una proteína de amplio rango SIGMA (C3437) (Bollag y Edelstein 1991). Los pesos moleculares de las proteínas se determinaron graficando en papel semilogarítmico las distancias de migración frente a los pesos moleculares de la proteína marcadora (Walker, 1984).

La resistencia a agentes antimicrobianos exhibida por las cepas de $V$. cholerae $\mathrm{O} 1$ estudia- das se muestra en la Tabla 1. Los antibióticos frente a los cuales la bacteria muestra mayor resistencia fueron $\mathrm{T}, \mathrm{Sx}$ y $\mathrm{S}(12,5 \%)$, lo cual indica una diferencia con respecto a lo reportado en 1991, donde se determinó la sensibilidad de $V$. cholerae O1 El Tor Inaba a la mayoría de antibióticos de uso farmacéutico (Tolmos, 1992). Se encontraron 4 cepas multirresistentes, destacando un aislado que presentó resistencia a T S

Tabla 1. Antibiograma de 32 cepas de Vibrio cholerae $\mathrm{O} 1$ aisladas de muestras clínicas durante el brote epidémico. Lima, 1998

\begin{tabular}{lrrrrrr}
\hline \multirow{2}{*}{ Antibiótico } & \multicolumn{2}{c}{ Sensibilidad } & \multicolumn{2}{c}{ Intermedio } & \multicolumn{2}{c}{ Resistente } \\
& $\mathrm{N}$ & $\%$ & $\mathrm{~N}$ & $\%$ & $\mathrm{~N}$ & $\%$ \\
\hline Tetraciclina & 28 & 87,5 & 0 & 0,0 & 4 & 12,5 \\
Cloramfenicol & 28 & 87,5 & 2 & 6,2 & 2 & 6,5 \\
Sulfatrimetoprim & 28 & 87,5 & 0 & 0,0 & 4 & 12,5 \\
Ampicilina & 21 & 65,6 & 10 & 31,3 & 1 & 3,1 \\
Estreptomicina & 14 & 43,7 & 14 & 43,7 & 4 & 12,5 \\
Ácido Nalidíxico & 25 & 78,1 & 5 & 15,6 & 2 & 6,2 \\
Furazolidone & 6 & 18,8 & 23 & 71,8 & 3 & 9,4 \\
Ciprofloxacina & 32 & 100,0 & 0 & 0,0 & 0 & 0,0 \\
Doxiciclina & 32 & 100,0 & 0 & 0,0 & 0 & 0,0 \\
Eritromicina & 5 & 15,6 & 25 & 78,1 & 2 & 6,2 \\
\hline
\end{tabular}

Tabla 2. Espectros de resistencia a antibióticos de uso comercial producidas por estirpes de Vibrio cholerae O1 aisladas de muestras clínicas. Lima, 1998

\begin{tabular}{llcc}
\hline Antibiotipo & $\begin{array}{c}\text { Espectro de resistencia } \\
\text { a antibióticos }(*)\end{array}$ & $\begin{array}{c}\text { Aislados } \\
\text { Clínicos }\end{array}$ & $\begin{array}{c}\text { Resistencia } \\
(\%)\end{array}$ \\
\hline 1 & TSCSxMFz & 1 & 3,1 \\
2 & TSCSx & 1 & 3,1 \\
3 & TSSx & 2 & 6,3 \\
4 & MFz & 1 & 3,1 \\
5 & A & 1 & 3,1 \\
6 & Fz & 1 & 3,1 \\
7 & EM & 2 & 6,3 \\
\hline
\end{tabular}

* $T$ = Tetraciclina (30); C = Cloramfenicol (30); $\mathrm{Sx}=$ Sulfatrimetoprim (25); $\mathrm{S}=$ Estreptomicina ( 10$)$; $\mathrm{A}=$ Ampicilina $(10) ; \mathrm{M}=$ Ácido Nalidixico (30); $\mathrm{Fz}=$ Furazolidone (100); E = Eritromicina (15) 


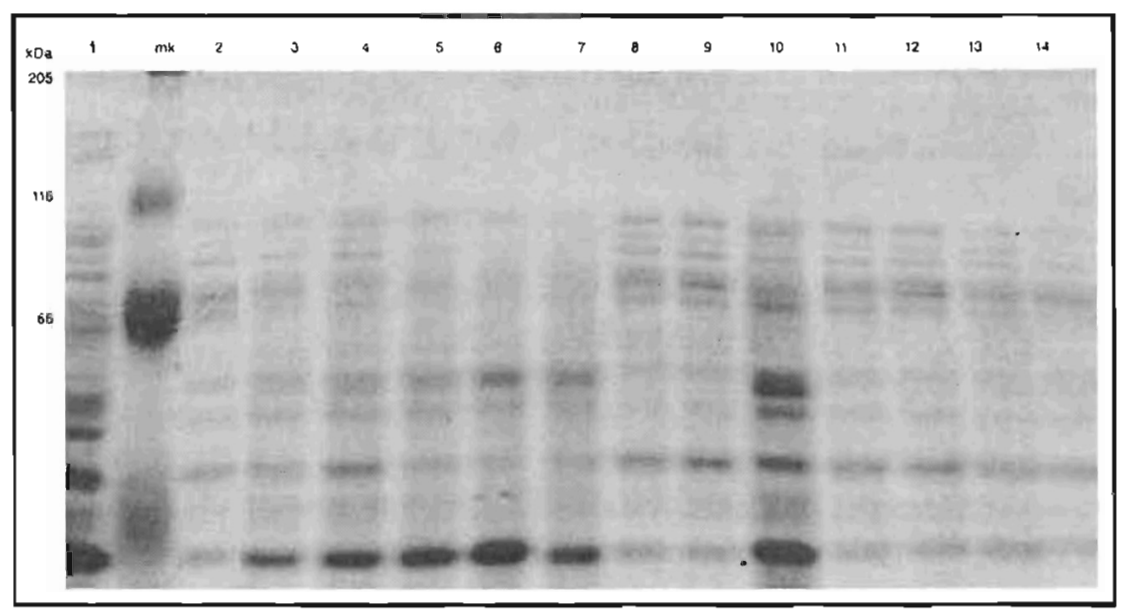

Figura 1. Perfiles protéicos de Aislados de Vibrio cholerae 01 $m K=$ Marcador; 1-4 y 6-14 V. cholerae Ogawa; $5 \mathrm{~V}$. cholerae Inaba; Resistencia antimicrobiana: 4 TSCSXMFz, 11 EM, 12 TSSx, 13 TSSx, $14 \mathrm{~A}$.

C Sx M y Fz $(3,1 \%)$. Se hallaron 7 antibiotipos siendo los más frecuentes: TSSx y $\operatorname{EM}(6,3 \%)$ (Tabla 2). Estos resultados muestran claramente la necesidad de realizar evaluaciones periódicas del espectro antimicrobiano: los hallazgos coinciden con estudios realizados previamente en Italia y Albania (Falbo et al., 1999).

Los perfiles proteicos de los diferentes aislados de $V$. cholerae $\mathrm{O} l$ estudiados fueron similares y estuvieron en un rango de 45 a $104,8 \mathrm{kDa}$ (Fig. 1). Las cepas Inaba y Ogawa presentaron el mismo patrón de bandas. Cabe destacar que las cepas con resistencia a los diferentes antimicrobianos utilizados presentaron perfiles proteicos similares en comparación a las que fueron sensibles. Debido a que los perfiles proteicos de las diferentes estirpes de $V$ cholerae $\mathrm{Ol}$ estudiadas presentaron un patrón homogéneo, no sería esta técnica una alternativa para el estudio de la evolución de este patógeno en relación a la resistencia a antimicrobianos y no brindaría información relevante que nos permita realizar un seguimiento periódico para lá vigilancia de cepas resistentes.

Los espectros de resistencia/sonsibilidad de los aislados de $V$. cholerae $\mathrm{Ol}$ del presente es- tudio a compuestos antimicrobianos son diferentes a los de brotes epidémicos anteriores ocurridos en nuestro pais, reportándose aislados clínicos multirresistentes en baja frecuencia. El esaudio de los perfiles proteicos no proporciona información relevante con relación a la resistencia a diferentes antibióticos utilizados en el tratamiento del cólera.

\section{LITERATURA GITADA}

Colwell,R.; Epstein, P.; Gubler, D.; Hall, M.; Reiter, P.; Shukla, J.; Sprigg, W.; Takajufi, E.; Trtanj, J. 1998. Global climate change and infections diseases. Emerg. Infect. Dis. 4(1).

Ibarra, J,; Delgado, A. y Alvarado, D. 1999. Vibrios no epidémico: y Vihrio cholercle Ol asociados a Enfermedad Diurreica Aguda Evento Climatológico "El Niño" 1998 - Hospitill Dos de Mayo. Rev. An. Fac. Med. 60: 251-56.

Gotuzzo, E.; Cieza, J.: Estremadoyro. L. and Seas, C. 1994. Cholera. Lessons from the Epidemic in Peru. In Isturiz \& Gotuzzo (ed.). Infectious Disedsc Clinics of North America. pp. 183-205. 
Seto, W.; Lau, F.; Gotuzzo, E. \& Carillo, C. 1992. Lomefloxacin versus Trimethoprim/ sulfamethoxazole in the treatment of adults with acute bacterial dianhea. Inter. Jour. Antimicro. Agent 2:61-66.

Seas, C. y Gotuzzo, E. 1999. Vibrio cholerae and other species. In Yu, V.; Merigan, T.; \& Barriere, $S$ (ed.). Antimicrobial Therapy and Vaccines. pp. 474-80.

Ibarra, J. y Alvarado, D. 1999. Evaluación de la resistencia a 2,4-Diamino-6,7-Diisopropilpteridina en estirpes de $V$. cholerae aisladas durante el ENOS 98 de muestras clínicas y ambientales. Resúmenes Seminario-Taller Red sobre impacto biológico de los Eventos "El Niño", Lima-Perú.

Bauer, A.; W. Kirby ; J. Sherris \& M. Turck. 1966. Antibiotic susceptibility testing by a standarized sing disk method. Am. J. Clin. Pathol. 45: 493-96.

National Committee for Clinical Laboratory Standards. 1992. Performance standards for antimicrobial disk susceptibility tests, $4^{\text {th }}$ ed. Approved standard. NCCLS document M100-54.

Bollag, D. \& Edelstein, S. (eds) 1991. Protein methods. WILEY-LISS, New York, USA, $230 \mathrm{pp}$.

Walker, J. 1984. Methods in molecular biology. Vol. 2. Nucleic Acids. The Human Press Inc. New Jersey.

Tolmos, J. 1992. Susceptibilidad antimicrobiana in vitro de cepas de Vibrio cholerae. Tesis Bachiller UPCH Lima, Perú, 31 pp.

Falbo, V.; Carattolli, A.; Tosini, F; Pezzella, C; Dionisi, A \& Luzzi, I. 1999. Antibiotic Resistence Conferred by a Conjugative Plasmid and a Class I Integron in Vibrio cholerae $\mathrm{OI} \mathrm{El}$ Tor Strains Isolated in Albania and Italy. Antim. Ag. Chemother. 43: 693-96. 